\title{
Estrategias basadas en coaching organizacional para incrementar calidad del talento humano en empresa de consultores
}

\author{
Strategies based on organizational coaching in order to increase quality of human \\ resources department in consultants company
}

\begin{tabular}{|c|c|c|}
\hline Viviana Aguilar & Antonio Márquez & Andreina Pérez \\
\hline $\begin{array}{l}\text { vivianaguilar16@hotmail.com } \\
\text { ORCID: 0000-0001-7405-9070 }\end{array}$ & $\begin{array}{l}\text { andreina.perez@hotmail.com } \\
\text { ORCID: 0000-0002-1350-6559 }\end{array}$ & $\begin{array}{l}\text { antoniomusk20@gmail.com } \\
\text { ORCID: 0000-0003-0916-4880 }\end{array}$ \\
\hline \multicolumn{3}{|c|}{ Universidad de Carabobo, Venezuela } \\
\hline
\end{tabular}

Artículo recibido en enero 2019 / Arbitrado en febrero 2019 / Publicado en mayo 2019

RESUMEN | Este estudio tuvo como objetivo general proponer estrategias basadas en el Coaching Organizacional para incrementar la calidad del talento humano de la empresa ARM Consultores, C. A. ubicada en la ciudad de Valencia, estado Carabobo, Venezuela ya que en la organización existe evidencia del bajo rendimiento del personal que impide el logro de los objetivos internos y la captación de nuevos clientes. Se diseñó una metodología basada en una investigación de campo, así como la aplicación de una encuesta como instrumento de recolección de datos. Los resultados obtenidos determinaron que la empresa debe promover la capacitación del personal como parte de sus funciones tales como Coaching Organizacional, gerencia estratégica, liderazgo organizacional entre otros. Se desarrolló una propuesta basada en estrategias de Coaching Empresarial, orientadas al acompañamiento del personal en un proceso no directivo que les permitiera aprovechar todos sus conocimientos, habilidades y destrezas.

Palabras clave: Coaching, organización, recurso humano, gerencia

\begin{abstract}
This study had as a general objective to propose strategies based on Organizational Coaching to increase the quality of the human talent of the company ARM Consultores C.A, located in the city of Valencia, Carabobo state, Venezuela, since in the organization there is evidence of low staff performance which prevents the achievement of internal objectives and the acquisition of new clients. A methodology based on a field investigation was designed, as well as the application of a survey as a data collection instrument. The results obtained determined that the company should promote staff training as part of its functions such as Organizational Coaching, strategic management, organizational leadership among others. A proposal was developed based on Business Coaching strategies, aimed at accompanying staff in a non-managerial process that allowed them to take advantage of all their knowledge, skills and abilities.
\end{abstract}

Keywords: Coaching, organization, human resources, management 


\section{INTRODUCCIÓN}

Hoy en día se pueden apreciar los grandes retos que enfrentan las organizaciones a nivel global. La rentabilidad de una empresa ha dejado de depender exclusivamente de la cantidad en cuanto a la producción de bienes o servicios y la calidad en todos los aspectos y procesos de la organización ha tomado mayor protagonismo en un entorno organizacional sumamente competitivo, de tal manera que un trabajador que tiene un alto desempeño en la calidad de su trabajo implica que está produciendo un servicio de calidad, pero cuando no ocurre así, evidentemente los resultados son adversos y las empresas de servicios se alejan cada vez más de sus objetivos, acarreando problemas como la ausencia de clientes que son la base fundamental en los ingresos de la empresa.

Actualmente, los líderes organizacionales reconocen esta situación y es por ello que optan por aplicar estrategias efectivas que les permitan potenciar su liderazgo e influenciar en sus colaboradores para poder gerenciar de manera eficiente. Tanto el llamado Coaching Organizacional como el Coaching Personal son consideradas estrategias 0 herramientas que brindan respuestas oportunas a la mejora del desempeño laboral.

Es por ello, que la complejidad y dinámica que caracteriza el entorno empresarial en el presente, ha sido influenciado por la globalización como fenómeno cultural y económico, por lo que se han agudizado la complejidad en las organizaciones, y por consiguiente, se han elevado los retos, a los cuales tienen que enfrentarse las organizaciones contemporáneas. Todo lo cual ha puesto de manifiesto con mayor énfasis la necesidad de hacer e incorporar transformaciones inmediatas en la forma de dirigir y gestionar las mismas, para garantizar el logro de los objetivos para los cuales fueron creadas. Situación que también está presente en el marco empresarial venezolano.

En este sentido, la realidad en muchas organizaciones es la falta de estrategias aplicadas por los líderes para promover un desempeño óptimo de su personal y que este cambio tenga incidencia en el éxito comercial de la organización; por tal motivo, Kilburg (2011), citado por Esparragoza y Palma (2012:24), comenta que "el reto de fomentar un equipo de trabajo eficiente y motivado comienza en la gerencia, pues son los líderes, quienes con las mejores prácticas gerenciales, pueden solucionar el problema de las deficientes relaciones entre directivos y colaboradores" y al mismo tiempo generar una ventana para el desarrollo organizacional, lo que se traduce en rendimiento económicos en el corto plazo.

En este orden de ideas, es pertinente considerar las ventajas que ofrece el coaching empresarial, para sentar las pautas de las prioridades organizacionales, como sistema interrelacionado, abierto y flexible. Así como también, buscar una concepción integradora y generadora de valor para los miembros de la organización. Dilts (2014:12) define al coaching organizacional como "El coaching es un camino para superar limitaciones. Permite hacer conscientes acciones, hábitos, valores, creencias, historias y juicios, a fin de facilitar procesos de cambio que permitan al cliente tomar acciones que lo lleven a ser una mejor persona, más completa".

A pesar de lo antes descrito y de los beneficios que ofrece el coaching empresarial como herramienta de desarrollo 
organizacional, muchos empresarios se han resistido a romper los esquemas tradicionales, con resultados poco efectivos para las organizaciones. Una de ellas ARM Consultores, C.A. empresa dedicada a la prestación del servicio de revisión fiscal, auditoría, asesorías tributarias, contables, financieras, de gestión empresarial, servicios externos de procesamiento electrónico de datos, análisis de proyecciones y estudios de factibilidad financiera, creada en el año 2014 y ubicada en la ciudad de Valencia, Estado Carabobo, objeto de este estudio.

Esta organización, a pesar de ser una empresa sólida y que la percepción de la gerencia es que sus empleados se sienten motivados desde el punto de vista económico, presenta debilidades en cuanto la capacidad de la organización de incorporar nuevos clientes; es por ello que con la presente investigación se busca observar a sus miembros para identificar aquellos factores determinantes para el desarrollo organizacional en la empresa y proponer la posterior aplicación del coaching como modelo para estimular el fortalecimiento comercial de la organización.

A través de una actividad consultiva realizada en la sede de la organización referida mediante entrevistas no estructuradas al personal, se han podido detectar los problemas que a continuación se exponen de forma sintetizada, destacando las más importantes: para empezar, se detecta la existencia entre los miembros del equipo de una visión a corto plazo de los planes, con énfasis en el cumplimiento de las metas técnicas y económicas, en contra del necesario pensamiento estratégico. Esto trae como consecuencia una escasa visión proactiva y excesiva operatividad en la solución de los problemas.
Existe además, un diseño estratégico no alineado totalmente con los procesos existentes, por lo cual los objetivos no se han convertido en estrategias ni se han aplicado indicadores de gestión para medir su eficacia y eficiencia, existe además una retroalimentación insuficiente sobre la mejora de los empleados, generando esto una disminución en la eficiencia y eficacia de su trabajo, detectándose la necesidad de crear mecanismos que se deben utilizar cuando un empleado de cualquier área merece ser felicitado por la ejecución ejemplar de alguna destreza, o cuando algún empleado de la organización necesita mejorar alguna habilidad dentro de su trabajo.

Por otra parte, en la empresa ARM Consultores, C.A. predomina la tradicional estructura por funciones, con énfasis en lograr mejores resultados en determinadas áreas. También se percibe cierta resistencia a la introducción de cambios que mejoran los procesos; también está presente la dirección centralizada respecto a la participativa; existen limitaciones en el enfoque de cliente interno, haciendo énfasis especialmente en el cliente externo, lo que trae como consecuencia deficiencias en la claridad respecto al alcance de los planes de desarrollo profesional de los trabajadores; insatisfacción de los clientes y trabajadores y una mala imagen respecto a la gestión de la calidad del recurso humano de la empresa.

En líneas generales, en la empresa ARM Consultores, C.A. el criterio de control y medición de los resultados del personal se orienta fundamentalmente hacia los indicadores tradicionales vinculados a la eficiencia de los resultados, respecto a aquellos vinculados a la efectividad. Aunque se debe destacar que existe un consenso 
bastante generalizado, por parte de los directivos de la empresa, de la necesidad de introducir un cambio estructural para dirigir y gestionar el capital humano de la misma. De hecho, muchos trabajadores han tomado conciencia de la importancia de la necesidad de incrementar sus habilidades, pero en la práctica aún persiste la resistencia al cambio y tienden a comportarse conservadoramente.

Tomando en cuenta estos aspectos, el presente trabajo de investigación tiene como objetivo general proponer estrategias basadas en el coaching organizacional para incrementar la calidad del talento humano de la empresa ARM Consultores, C. A. ubicada en la ciudad de Valencia, estado Carabobo, Venezuela, con la finalidad de que esta empresa pueda incrementar la calidad de su servicio y atraer mayor número de clientes. En vista de lo planteado, se debe dar respuesta a la siguiente interrogante: ¿Cuáles elementos han de considerarse para proponer un estrategias basadas en las herramientas del coaching empresarial que contribuya al incremento de la calidad del recurso humano de la empresa ARM Consultores, C.A., ubicada en Valencia, estado Carabobo?

\section{MATERIALES Y METODOS}

El tipo de investigación de este estudio corresponde a una investigación cualitativa. Por lo tanto, se recogen y analizan datos cualitativos sobre variables, basadas en las percepciones de los participantes, utilizando para ellos métodos como la encuesta o la entrevista.

En este sentido, la naturaleza cualitativa de la presente investigación radica en el hecho de que las variables y los indicadores derivados de éstas son evaluados a través de los puntos de vistas $u$ opiniones obtenidos por medio del instrumento de recolección de información seleccionado para la investigación.

Del mismo modo, desde el nivel comprensivo, la investigación se comprende dentro de la modalidad de un proyecto factible, en el diseño de una propuesta de acción dirigida a resolver un problema o necesidad previamente detectada en el medio.

\section{RESULTADOS Y DISCUSION}

En relación con los resultados obtenidos y la creación de un ambiente de trabajo idóneo para el trabajador, Mintzberg (2008) comenta que el área responsable de administrar las relaciones laborales regularmente es la gerencia o dirección de recursos humanos, su responsabilidad directa es el propiciar las relaciones armoniosas y de cooperación de ambas partes como una estrategia para obtener el máximo de beneficios para las dos partes involucradas. De esta forma, poseer buena moral y actitud en el lugar de trabajo, contribuye al rendimiento del empleado, la cooperación entre ellos, desarrollo profesional de los buenos trabajadores y por ende el funcionamiento efectivo de la empresa.

En relación con la experiencia provista por el desempeño laboral, Chiavenato (2003), indica que el concepto de experiencia, dentro de la formación de competencias, surge de la necesidad de valorar no sólo el conjunto de los conocimientos apropiados (saber) y las habilidades y destrezas (saber hacer) desarrolladas por una persona, sino de apreciar su capacidad de emplearlas para responder a situaciones, resolver problemas y desenvolverse en el mundo. Igualmente, implica una mirada a las condiciones del 
individuo y disposiciones con las que actúa, es decir, al componente actitudinal y valorativo (saber ser) que incide sobre los resultados de la acción.

Por otra parte, de acuerdo con Alvarado (2008), el respeto es uno de los componentes que identifica que el empleado está seguro en su empleo. Esta seguridad es proporcionada por la organización que muestra que está comprometida con su fuerza de trabajo. Según estudios hechos por el autor, hay investigaciones que muestran la relación entre la seguridad del empleado en el empleo con la confianza en la gestión, reducción de los niveles de rotación de personal y el desempeño de la organización.

Lo relacionado con la participación del personal como estrategia de satisfacción dentro de los modelos de coaching, Bohnenberger (2005), indica que la participación del personal consiste en el aprovechamiento de conocimientos, habilidades $\mathrm{y}$ actitudes que aplicadas $\mathrm{o}$ demostradas en situaciones del ámbito productivo, tanto en un empleo como en una unidad para la generación de ingreso por cuenta propia, se traducen en resultados efectivos que contribuyen al logro de los objetivos de la organización o negocio.

En otras palabras, la competencia laboral es la capacidad que una persona posee para desempeñar una función productiva en escenarios laborales usando diferentes recursos bajo ciertas condiciones, que aseguran la calidad en el logro de los resultados. Por otro lado, el conocimiento de las necesidades de los empleados permite al sector de recursos humanos desarrollar actividades para atenderlas de forma eficaz. Es preciso recordar que en tiempos de marketing de relación, es necesaria la adecuación de todos los procesos para dejar el cliente satisfecho y conectado con la organización y para coaching empresarial no es diferente.

Asimismo, como actividad específica del proceso de comunicación interna, Rafiq y Ahmed (2010), propone la difusión de objetivos y metas de la organización. Según el autor, los empleados deben no solo conocer los caminos de la organización, como también analizarlos y sugerir cambios cuando posible. Asimismo, se debe dar a conocer los resultados alcanzados y la situación de la organización, ya que representan la información referente al nivel de desarrollo de los sectores y de la organización como un todo. Además, puede estar asociada directamente a un programa de reconocimiento formal y tiene como ventaja el conocimiento de los empleados de la situación real de su organización que proporciona una cierta seguridad en el empleo.

\section{La Propuesta}

\section{Título de la Propuesta}

Estrategias basadas en las herramientas del coaching empresarial orientado al incremento de la calidad del recurso humano de la empresa ARM Consultores, C. A.

\section{Presentación}

El propósito fundamental de la propuesta consiste en proveer a la empresa ARM Consultores, C. A. de un plan de actividades que conduzcan a la implementación del coaching empresarial, como herramienta útil para el incremento de su calidad y el mejoramiento de sus resultados financieros y organizacionales. De esta forma, la propuesta contiene el establecimiento claro de los objetivos que se buscan alcanzar a 
través del programa, lo cual se justifica ante la necesidad del coaching empresarial que ayude a los líderes a desarrollar mejor sus funciones y a lograr una eficiente labor en sus empleados.

\section{Fundamentación}

Al realizar el diagnóstico situación actual de la gestión del recurso humano de la empresa ARM Consultores, C. A. y la identificación de los factores claves de la gestión de recursos humanos de la empresa, se determinó la necesidad en la empresa de implementar un modelo de coaching empresarial como técnicas modernas de liderazgo, que permita a los líderes de la organización actualizar sus conceptos teóricos y prácticos que conlleven a un proceso organizado para lograr el incremento de la calidad del recurso humano, mediante un proceso de aprendizaje que consiste en una relación profesional en la que un entrenador o facilitador guía y estimula a una o más personas con el objetivo de motivar su crecimiento y el desarrollo de su pleno potencial, lo que les permitirá alcanzar el éxito profesional y el logro de sus metas personales.

En cuanto a su aplicación, el programa de coaching empresarial está concebido exclusivamente para las personas con posiciones de liderazgo en la organización, el cual permitirá aportar calidad al recurso humano, con la intención de maximizar el desempeño en sus actividades laborales. Se pretende que estas personas sumen nuevas posturas de liderazgo, e incorporen a nuevos trabajadores al proceso como parte de un plan de desarrollo de carrera, beneficiando así al desarrollo de los empleados. Del mismo modo, el programa propuesto tratará de facilitar e incorporar nuevas herramientas para el trabajo de líderes al momento de dirigir a sus empleados; ya que por naturaleza el coaching pretende crear una comunicación fluida entre el líder $y$ empleado.

\section{Objetivos de la Propuesta}

\section{Objetivo General}

Proponer estrategias basadas en las herramientas del coaching empresarial orientado al incremento de la calidad del recurso humano de la empresa ARM Consultores, C. A.

\section{Objetivos Específicos}

Estructurar un modelo de Coaching Empresarial, que esté de acuerdo a los recursos y necesidades de la empresa ARM Consultores, C. A.

Describir cada una de las etapas y actividades necesarias para desarrollar el modelo de coaching empresarial en la empresa ARM Consultores, C. A.

\section{Beneficios de la propuesta}

Al momento de evaluar los beneficios del programa propuesto, hay que mencionar que impacta directamente sobre los resultados de la empresa, ya que en la medida que los mercados van evolucionando y se vuelven más competitivos; las organizaciones se encuentran ante nuevos retos, obligándolos a tener una visión más agresiva de las medidas que puedan adoptar para apoderarse de una porción del mercado.

En este sentido, algo importante de mencionar es que las empresas en el área de servicios no solamente necesitan la tecnología más adecuada para su desarrollo, sino que también necesitan recurso humano capacitado que contribuyan al logro de los 
objetivos de la organización. Es por eso que el modelo del coaching empresarial desarrollará integralmente el desempeño de los empleados, incrementando sus capacidades de desenvolverse eficientemente en el campo de acción

\section{Desarrollo de la Propuesta}

La gerencia general de la empresa ARM Consultores, C. A., siempre está en busca de alternativas que la hagan competitiva; por ello, entre las opciones disponibles, elige siempre la que vaya más acorde a sus necesidades. Para tomar estas decisiones la organización debe disponer de información y debe escoger un modelo para desarrollar y utilizar esta información, la cual variará en forma directamente proporcional con el tamaño de la misma.

Diseño del Plan General del Programa de Coaching Empresarial

Después de haber analizado los resultados del instrumento de recolección de información aplicado en la empresa ARM Consultores, C. A., se plantea en esta etapa el plan general del programa de coaching empresarial para los líderes y empleados, concluyendo en la necesidad de implementar este modelo. En esta etapa se plantean los objetivos, las estrategias y políticas que serán utilizadas para el desarrollo y aplicación del programa de coaching empresarial, ya que utilizando este modelo puedan lograr un desempeño eficiente e incrementar la calidad de su recurso humano. En la figura 1 se muestra una representación de cada una de las etapas y actividades necesarias para cumplir con las estrategias basadas en las herramientas del coaching empresarial orientado al incremento de la calidad del recurso humano de la empresa ARM Consultores, C. A. 


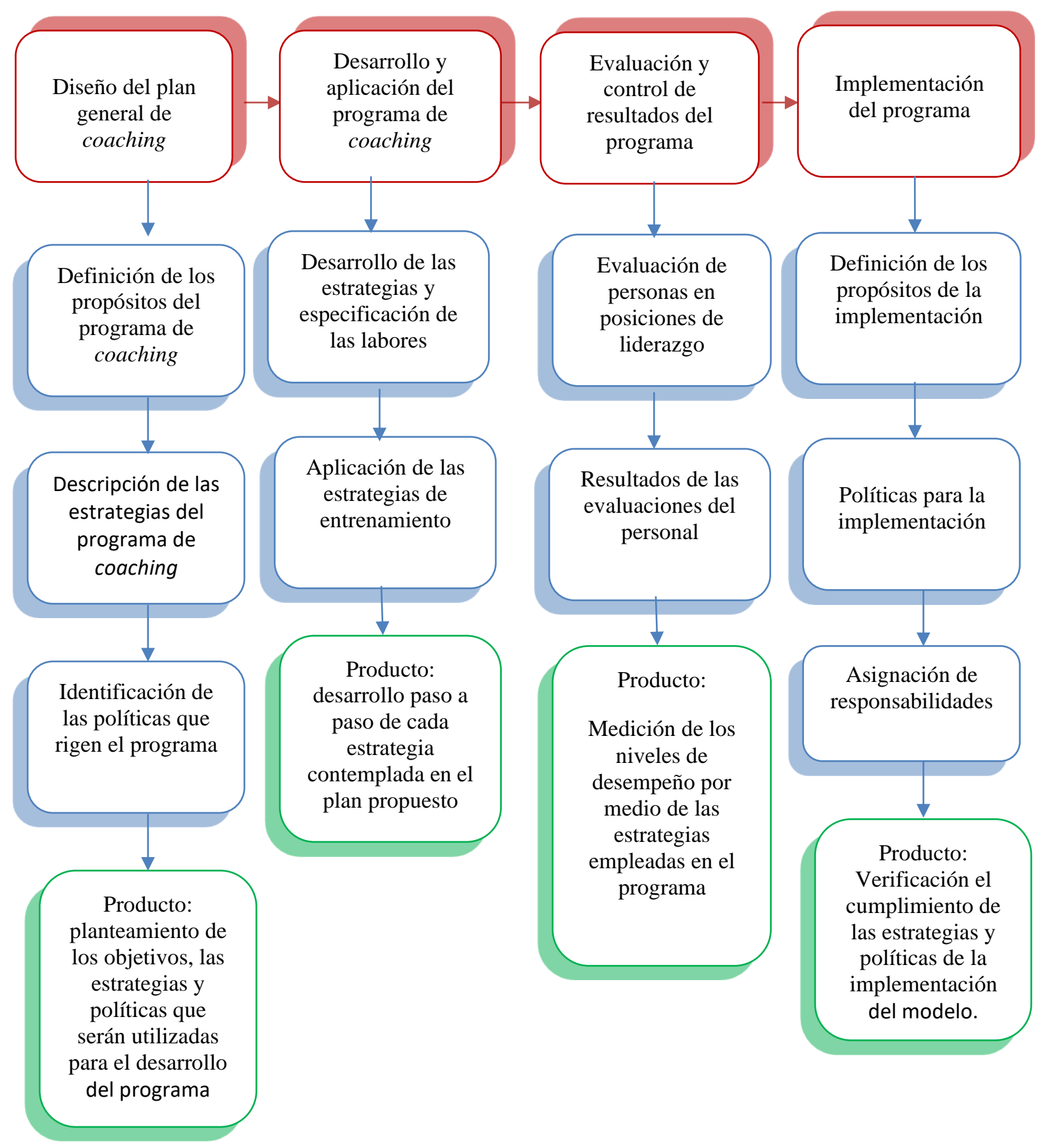

Figura 1. Plan de acción para aplicar las estrategias basadas en las herramientas del coaching empresarial orientado al incremento de la calidad del recurso humano de la empresa ARM Consultores, C. A.

Fuente: Aguilar, Márquez y Pérez (2017). 
CONCLUSIONES

Las organizaciones de hoy en día están siendo fuertemente influenciadas por las diversas tendencias que rigen el mundo empresarial como por ejemplo las ventas, el marketing, la manufactura, los clientes entre otros. No obstante hay un factor que no varía en el tiempo y que sin duda es el pilar fundamental de toda organización ya que sin este, las empresas son cuerpos vacíos incapaces de crecer por sí mismas, nos referimos específicamente al talento humano; factor de gran relevancia, aún más en las empresas dedicadas al servicio, ya que una compañía que se dedique al servicio de terceros y que carezca de un buen desempeño laboral por parte de sus colaboradores es muy probable que este limitada a la obtención de sus objetivos.

En vista de ello, la presente investigación tuvo como objetivo general proponer estrategias basadas en el coaching organizacional para incrementar la calidad del talento humano de la empresa ARM Consultores, C. A. ubicada en la ciudad de Valencia, estado Carabobo., para lo cual se propusieron tres objetivos específicos, los cuales en forma organizada y sistematizada, contribuyeron al logro de las metas del estudio, y de los cuales se extraen las siguientes conclusiones:

Inicialmente se definió el primer objetivo cuyo propósito consistió en diagnosticar la situación actual en la gestión del recurso humano de la empresa ARM Consultores, C. A., para la identificación de las oportunidades de mejora. Al respecto se obtuvieron las siguientes conclusiones: En una empresa dedicada a la prestación de servicios profesionales el buen desempeño del trabajador está vinculado con la buena calidad en el servicio.
Es deber del líder del equipo o de la gerencia de la empresa, promover las estrategias planteadas para la obtención de los objetivos de la organización. Sin embargo los líderes organizacionales en ciertas ocasiones están involucrados exclusivamente en los procesos relacionados a la operatividad de la empresa, por lo que descuidan la calidad del talento humano y es allí donde comienzan a aparecer focos de bajo desempeño y por lo tanto los resultados no son los deseados

Tomando en consideración esto, los líderes o gerentes de las empresas que prestan servicios profesionales comprenden que es necesaria la aplicación de nuevas estrategias que se orienten a fortalecer el desempeño del personal y que este pueda cumplir con las metas pautadas por la organización a largo plazo.

Luego, el segundo de los objetivos especíicos fue Identificar los factores claves en la gestión del recurso humano que deben formar parte de un programa de coaching empresarial. Al respecto, se concluye que la empresa debe reconocer las debilidades que posee y tomar conciencia de ellas para enfrentarlas con un nuevo enfoque.

También se concluye que es de vital importancia la medición constante de indicadores en cuanto al cumplimiento de las metas que no solo estén relacionados al rendimiento en la operatividad sino también al desempeño del trabajador es la mejor manera que la empresa tiene para identificar sus debilidades y fortalezas en cuanto al cumplimiento de sus objetivos.

Con la información obtenida se procedió a diseñar un programa de coaching organizacional aplicado en un grupo de diez personas en las que se incluyó a los dos líderes del equipo en la empresa ARM 
Consultores, C.A. El programa de coaching organizacional constituye una herramienta para armonizar lo operativo con el desempeño eficiente del personal, con lo cual se contribuye con uno de los objetivos de la organización que es poder ampliar su espectro con relación a la cantidad de clientes a la que aspira poder ofrecer sus servicios en el corto, mediano y largo plazo, para ello se elaboró una propuesta con la que se persigue: Proveer en la empresa ARM Consultores, C. A. Un plan de actividades que conduzcan a la aplicación del Coaching Empresarial, como instrumento útil para el incremento en la calidad del servicio y el mejoramiento de sus resultados financieros y organizacionales.

De esta forma, la propuesta establece los objetivos que se buscan obtener a través del programa de coaching organizacional, con lo que se busca acompañar a los líderes en el proceso de autodescubrimiento para desarrollar mejor sus funciones y a lograr una mayor influencia en sus colaboradores.

\section{REFERENCIAS}

Arias, Fidias (2012) El proyecto de Investigación, Introducción a la metodología científica. Quinta Edición. Episteme, Caracas, Venezuela

Balestrini, Miriam (2008). Cómo se elabora el Proyecto de Investigación para los estudios formulativos 0 exploratorios, descriptivos, diagnósticos, evaluativos, formulación de hipótesis causales, experimentales y los proyectos factibles. Sexta Edición. BL Consultores Asociados. Servicio Editorial. Caracas

Caicedo, Lina, Herrera, José Ricardo y Quenguán, Natalia (2013). El coaching como herramienta para el desarrollo de los recursos humanos en la empresa. Trabajo de grado no publicado. Universidad del Rosario (España)
Documento en línea. Recuperado: noviembre 2016. Disponible en:

http://repository.urosario.edu.co/bitstream/ handle/10336/4781/10752497122013.pdf? sequence $=1$

Chiavenato, Idalberto (2009). Administración de Recursos Humanos. Editorial McGraw Hill, Ciudad de México

Chirinos, A. Colina M. y Rojas, Y. (2012). Coaching gerencial como herramienta de gestión de la empresa Hidra-Lite ubicada en el municipio Valencia, Estado Carabobo. Trabajo de grado no publicado. Universidad de Carabobo (UC), Bárbula

Dilts, Robert (2012). Coaching: herramientas para el cambio. Editorial Urano, Barcelona, España

Esparragoza, G. y Palma, A. (2012). Estudio del coaching sobre los niveles de capital intelectual del personal que labora en una empresa dedicada al ramo papelero en el estado Carabobo. Trabajo de Grado no publicado. Universidad de Carabobo, Bárbula

González, Luis. (2010). Técnicas de gestión de recursos humanos. Revista de Psicología General y Aplicada, 43, Madrid, España

González, Y. Hernández, L. y Tarazona, G. (2010). El coaching como herramienta para la formación del gerente creativo. Trabajo de Grado no publicado. Universidad de Carabobo, Bárbula

Hurtado, Jacqueline. (2008). El proyecto de investigación. Comprensión holística de la metodología y la investigación. Sexta edición. Quirán Ediciones, Caracas

Hurtado, Iván y Toro, Josefina (2010). Paradigmas y métodos de investigación en tiempos de cambio. Quinta Edición. Episteme Editores. Valencia, Venezuela

Kilburg, Richard (2011). Executive coaching: Developing managerial wisdom in a world of chaos (Coaching ejecutivo: desarrollo de la sabiduría gerencial en un mundo de caos). Publicaciones de la American 
Psychological Association, Washington, EE.UU

Lozano, Luz Janeth (2014). El coaching como estrategia para la formación de competencias profesionales. Artículo publicado en la Revista Institucional de la Universidad EAN, Bogotá (Colombia). Documento en línea. Recuperado: noviembre 2016. Disponible en:

http://www.redalyc.org/articulo.oa?id=2061 1455009

Mintzberg, Henry (2010). Diseño de Organizaciones Eficientes. Mc GrawHill de Management, Ciudad de México

Muradep. Lidia (2014). Coaching para la trasformación personal. Editorial Management Comunicación Granica. Buenos Aires, Argentina

Ortiz, E. y Ruiz, J. (2011). Estrategias basadas en la teoría del coaching para el mejoramiento productivo de los empleados Caso: firma contable MBC y Asociados. Trabajo de Grado no publicado. Universidad de Carabobo, Bárbula

Sabino, Carlos (2010) El Proyecto de Investigación. Caracas: Ediciones Panapo.

Scheim, Edgar (2008). Cultura organizacional y liderazgo. Jossey Bass Publishers, San Francisco, EE.UU

Tamayo y Tamayo, Mario (2012). El Proceso de la Investigación Científica. 4⿳a Edición. Editorial Limusa S.A

Vargas, Fernando (2016). La formación por competencias: Instrumento para incrementar la empleabilidad. Artículo publicado en el volumen 9 de la revista de la Escuela de Comunicación Lingüística de la Universidad Nacional de Bogotá, Colombia 